\title{
Formation of organically modified octacalcium phosphate in solutions containing various amounts of benzenedicarboxylic acids
}

\author{
Taishi YOKOI, ${ }^{\dagger}$ Masanobu KAMITAKAHARA, ${ }^{*}$ Masakazu KAWASHITA ${ }^{* *}$ and Chikara OHTSUKI \\ Department of Crystalline Materials Science, Graduate School of Engineering, Nagoya University, \\ Furo-cho, Chikusa-ku, Nagoya 464-8603, Japan \\ ${ }^{*}$ Graduate School of Environmental Studies, Tohoku University, 6-6-20 Aoba, Aramaki, Aoba-ku, Sendai 980-8579, Japan \\ ${ }^{* *}$ Department of Biomedical Engineering, Graduate School of Biomedical Engineering, Tohoku University, \\ 6-6-11-1306-1 Aramaki-Aoba, Aoba-ku, Sendai 980-8579, Japan
}

\begin{abstract}
Octacalcium phosphate (OCP) has a layered structure composed of apatitic and hydrated layers. $\mathrm{HPO}_{4}{ }^{2-}$ in the hydrated layer can be substituted by dicarboxylate ions. We investigated formation of OCP with incorporated dicarboxylate ions, namely octacalcium phosphate carboxylate (OCPC), under synthetic conditions with various concentrations of benzenedicarboxylic acids (BDCAs) such as 1,2-benzenedicarboxylic acid (1,2BDCA, phthalic acid), 1,3-benzenedicarboxylic acid (1,3BDCA, isophthalic acid), and 1,4-benzenedicarboxylic acid (1,4BDCA, terephthalic acid). 1,3BDCA or a small amount of 1,2BDCA could be incorporated into $\mathrm{OCP}$, but 1,4BDCA could not be incorporated into $\mathrm{OCP}$. When the added amount of 1,3BDCA was larger than 2 mmol, OCPC with 1,3BDCA (1,3BDCA-OCPC) was formed with dicarboxylate-free OCP (Pure-OCP). The fractions of 1,3BDCA-OCPC with respect to Pure-OCP increased with increasing amounts of 1,3BDCA. When excessive amounts of 1,3BDCA, namely 25 times with respect to the stoichiometric composition of OCPC, were used for formation of OCPC, 1,3BDCAOCPC was formed as a single phase. Addition of excessive amounts of 1,3BDCA prevented formation of Pure-OCP and had an advantage in formation of 1,3BDCA-OCPC.
\end{abstract}

(C2013 The Ceramic Society of Japan. All rights reserved.

Key-words : Octacalcium phosphate, Benzenedicarboxylic acids, Organic modification, Substitution, Wet chemical process

[Received October 5, 2012; Accepted November 29, 2012]

\section{Introduction}

Organically modified calcium phosphates are interesting materials. Phosphoric acid has four chemical states, namely $\mathrm{H}_{3} \mathrm{PO}_{4}, \mathrm{H}_{2} \mathrm{PO}_{4}{ }^{-}, \mathrm{HPO}_{4}{ }^{2-}$, and $\mathrm{PO}_{4}{ }^{3-}$. Hence phosphoric acid can form salts with calcium ions in various ratios. Hydroxyapatite [HAp: $\left.\mathrm{Ca}_{10}\left(\mathrm{PO}_{4}\right)_{6}(\mathrm{OH})_{2}\right]$ is a typical calcium phosphate used as a ceramic biomaterial, especially as artificial bone. $\alpha$ - and $\beta$-tricalcium phosphates [TCP: $\mathrm{Ca}_{3}\left(\mathrm{PO}_{4}\right)_{2}$ ] have been investigated as bone-repairing materials with biodegradability. $\alpha$-TCP can be transformed into the other calcium phosphate compounds, such as HAp and octacalcium phosphate [OCP: $\left.\mathrm{Ca}_{8}\left(\mathrm{HPO}_{4}\right)_{2}\left(\mathrm{PO}_{4}\right)_{4} \cdot 5 \mathrm{H}_{2} \mathrm{O}\right] .{ }^{1)-5)}$ Dicalcium phosphate anhydrous (DCPA: $\mathrm{CaHPO}_{4}$ ) and dicalcium phosphate dihydrate (DCPD: $\mathrm{CaHPO}_{4} \cdot 2 \mathrm{H}_{2} \mathrm{O}$ ) are typical calcium phosphate compounds that can be synthesized in a solution under normal pressure and temperature conditions. ${ }^{6}$ ) Among these calcium phosphates, OCP has a crystallographically unique property: a layered structure composed of apatitic and hydrated layers. ${ }^{7)}$ The layered structure is constructed by $\mathrm{HPO}_{4}{ }^{2-}$, which plays the part of a pillar in the hydrated layer. The $\mathrm{HPO}_{4}{ }^{2-}$ links two apatitic layers. The $\mathrm{HPO}_{4}{ }^{2-}$ ions in the hydrated layer can be substituted by dicarboxylate ions. ${ }^{8)}$ OCP with incorporated dicarboxylate ion is called OCP carboxylate (OCPC). The general chemical formula of OCPC is $\mathrm{Ca}_{8}\left(\mathrm{HPO}_{4}\right)_{2-z}(\mathrm{DCI})_{z}\left(\mathrm{PO}_{4}\right)_{4} \cdot m \mathrm{H}_{2} \mathrm{O}$, where

Corresponding author: T. Yokoi; E-mail: yokoi.taishi@a.mbox. nagoya-u.ac.jp
DCI refers to dicarboxylate ion. ${ }^{8)}$ The interplanar spacing of the $(100)$ planes $\left(d_{100}\right)$ of OCP is expanded by incorporation of dicarboxylate ions into the OCP. For example, the $d_{100}$ value of dicarboxylate-free OCP is $1.87 \mathrm{~nm}$, while the $d_{100}$ values of OCPC with succinate ion (Suc-OCPC) and suberate ion (SubOCPC) are 2.14 and $2.61 \mathrm{~nm}$, respectively. ${ }^{9)}$

OCPCs have a potential to show various properties depending on the dicarboxylate ions incorporated. Various kinds of dicarboxylic acids can be incorporated into OCP crystals, hence we can expect that OCPCs show selective adsorption properties depending on the sizes, side groups and polarities of the dicarboxylate ions incorporated into OCP. For example, Aoki et al. reported a specific adsorption of an aldehyde for OCPC with aspartate ion. ${ }^{10)}$

Several methods for the synthesis of OCPCs have been reported. Typically, OCPC is synthesized by the hydrolysis of $\alpha$-TCP in a dicarboxylic acid solution. ${ }^{11)-13)}$ Monma reported that OCPCs with aliphatic dicarboxylate ions and benzenedicarboxylate ions were synthesized from $\alpha$-TCP. ${ }^{14)}$ He reported that both 1,2-benzenedicarboxylic acid (1,2BDCA, phthalic acid) and 1,3-benzenedicarboxylic acid (1,3BDCA, isophthalic acid) were incorporated into OCP, while 1,4-benzenedicarboxylic acid (1,4BDCA, terephthalic acid) was not incorporated into OCP. However, detailed characterizations for OCPC with benzenedicarboxylate ions (BDCAs-OCPC) such as X-ray diffraction and Fourier-transform infrared measurements were not reported. The fundamental findings on formation and structure of BDCAsOCPC are useful to develop OCPC-based materials. For example, BDCAs have a benzene ring on their structures, hence 
we expect that BDCAs-OCPC will show a selective adsorption property for hydrophobic volatile organic compounds, such as benzene and toluene.

It is desirable to evaluate the properties of crystalline materials that have high crystallinity and high purity. Kamitakahara et al. reported that the crystallinity of OCPCs synthesized by using calcium carbonate $\left(\mathrm{CaCO}_{3}\right)$, phosphoric acid $\left(\mathrm{H}_{3} \mathrm{PO}_{4}\right)$, and dicarboxylic acids was higher than that synthesized through the hydrolysis of $\alpha$-TCP in a dicarboxylic acid solution (e.g., SucOCPC, OCPC with adipate ion (Adi-OCPC), and Sub-OCPC). $\left.{ }^{15}\right)$ Additionally, we reported that the fractions of Suc-OCPC and Sub-OCPC in samples increased with increasing concentrations of Suc and Sub in the reaction solution. ${ }^{16)}$ In this synthesis method, the formation of Suc-OCPC and Sub-OCPC required excessive amounts of dicarboxylic acids (over 10 times with respect to the stoichiometric composition of OCPC) for the formation of OCPCs as single phases. Hence formation of BDCAs-OCPC with high crystallinity and high purity is expected through the process using $\mathrm{CaCO}_{3}, \mathrm{H}_{3} \mathrm{PO}_{4}$, and excessive amounts of BDCAs. However, synthesis of BDCAs-OCPC by using $\mathrm{CaCO}_{3}, \mathrm{H}_{3} \mathrm{PO}_{4}$, and BDCAs, namely 1,2BDCA, 1,3BDCA and 1,4BDCA, has not been attempted and the relationship between concentrations of these BDCAs in the reaction mixture and the fraction of BDCAs-OCPC in obtained samples were still unclear. In the present study, we investigated formation of OCPCs in a solution containing various amounts of 1,2BDCA, 1,3BDCA, and 1,4BDCA. We selected the concentration of BDCAs in the reaction solutions as an experimental parameter, because the concentration of dicarboxylic acids in a reaction solution is one of the dominant factors for formation of OCPC.

\section{Experimental procedures}

\subsection{Formation of OCPCs under presence of benzenedicarboxylic acids}

Various amounts of 1,2BDCA, 1,3BDCA, or 1,4BDCA (Wako Pure Chemical Industries Ltd., Osaka, Japan) were added in $100 \mathrm{~cm}^{3}$ of ultrapure water. The $\mathrm{pH}$ of the solutions was adjusted to 5.5 by addition of ammonia solution $\left(\mathrm{NH}_{3} ; 25 \%\right.$ aqueous solution, Wako Pure Chemical Industries Ltd.). The amounts of BDCAs were $0,0.50,1.0,2.0,5.0,10$ and $25 \mathrm{mmol}$. Figure 1 shows the molecular structures of 1,2BDCA, 1,3BDCA, and 1,4BDCA. These dicarboxylic acids have two carboxyl groups on the benzene ring. The carboxyl groups in 1,2BDCA, 1,3BDCA, and 1,4BDCA are in the ortho, meta, and para positions, respectively. The dissociation constants of these BDCAs are the following: $1,2 \mathrm{BDCA}, \mathrm{p} K_{\mathrm{a} 1}=2.9$ and $\mathrm{p} K_{\mathrm{a} 2}=5.5 ; 1,3 \mathrm{BDCA}, \mathrm{p} K_{\mathrm{a} 1}=$ 3.5 and $\mathrm{p} K_{\mathrm{a} 2}=4.6$; and $1,4 \mathrm{BDCA}, \mathrm{p} K_{\mathrm{a} 1}=3.5$ and $\left.\mathrm{p} K_{\mathrm{a} 2}=4.8 .{ }^{17}\right)$ $8.0 \mathrm{mmol}$ of $\mathrm{CaCO}_{3}$ (calcite; Nacalai Tesque Inc., Kyoto, Japan) was suspended in the dicarboxylic acid solution. A $5.0 \mathrm{mmol}$ of $\mathrm{H}_{3} \mathrm{PO}_{4}$ (85\% aqueous solution, Nacalai Tesque Inc.) was added to the suspension. The suspension was stirred at $60^{\circ} \mathrm{C}$ for $6 \mathrm{~h}$. The precipitates were isolated by suction filtration then dried at $40^{\circ} \mathrm{C}$ overnight. The synthesis procedure is shown in Fig. 2.

Dicarboxylate-free OCP was synthesized by mixing $8.0 \mathrm{mmol}$ of $\mathrm{CaCO}_{3}$ and $6.0 \mathrm{mmol}$ of $\mathrm{H}_{3} \mathrm{PO}_{4}$ in $100 \mathrm{~cm}^{3}$ of ultrapure water at $60^{\circ} \mathrm{C}$ for $6 \mathrm{~h}$ as previously reported. ${ }^{15)}$ Hereafter dicarboxylatefree OCP is abbreviated as Pure-OCP.

\subsection{Characterization}

The obtained samples were characterized by powder X-ray diffraction (XRD; RINT PC2100, Rigaku Co., Tokyo, Japan) using $\mathrm{Cu} \mathrm{K} \alpha$ radiation $(\lambda=0.154056 \mathrm{~nm})$. The crystalline phases of the samples were examined by powder XRD in the range $2 \theta=2-40^{\circ}$ with a scanning rate of $1.0^{\circ} \mathrm{min}^{-1}$. The composition of a sample was determined using by inductively coupled plasma atomic emission spectroscopy (ICP-AES: Optima 2000DV, PerkinElmer Japan, Yokohama, Japan) following dissolution of $1 \mathrm{mg}$ precipitate in a $20 \mathrm{~cm}^{3}$ of $5 \mathrm{vol} \%$ nitric acid solution. The chemical structures of the representative samples were characterized using Fourier-transform infrared (FTIR) spectroscopy (FT/IR-6100; JASCO Co., Tokyo, Japan), using the KBr tablet method. The mass ratio of $\mathrm{KBr}$ to sample was 100:1. Each sample was scanned between 4000 and $400 \mathrm{~cm}^{-1}$ at a resolution of $4 \mathrm{~cm}^{-1}$. The morphologies of the precipitates were observed by scanning electron microscopy (SEM; JSM5600, JEOL Ltd., Tokyo, Japan), after application of a thin gold coating.

To examine the formation process and transformation behaviors of OCPs, sample powders were obtained, after addition of $\mathrm{H}_{3} \mathrm{PO}_{4}$, by suction filtration of $2 \mathrm{~cm}^{3}$ aliquots of reaction mixtures

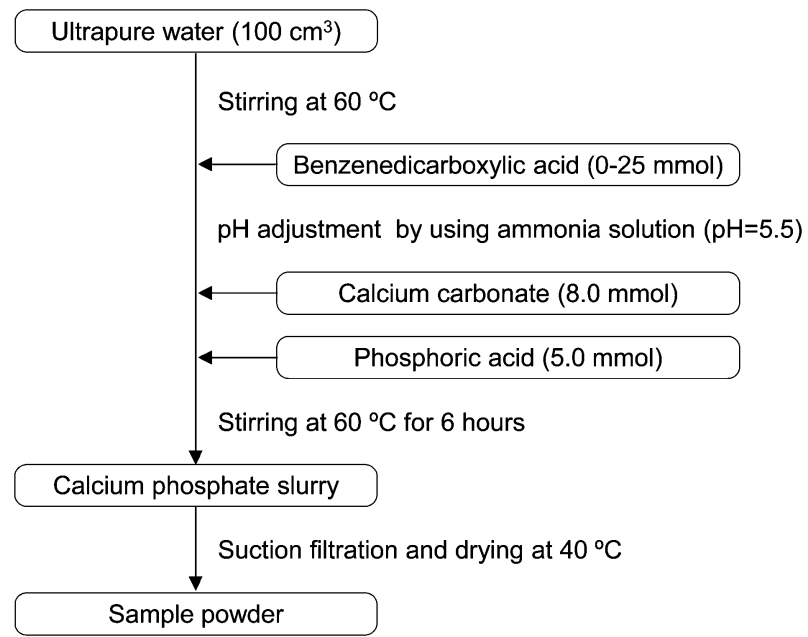

Fig. 2. Procedures for preparing OCPC with benzenedicarboxylate ions.<smiles>O=C(O)c1ccccc1C(=O)O</smiles><smiles>O=C(O)c1cccc(C(=O)O)c1</smiles><smiles>O=C(O)c1ccc(C(=O)O)cc1</smiles>

Fig. 1. Molecular structures of (a) 1,2-benzenedicarboxylic acid (1,2BDCA, phthalic acid), (b) 1,3-benzenedicarboxylic acid (1,3BDCA, isophthalic acid) and (C) 1,4-benzenedicarboxylic acid (1,4BDCA, terephthalic acid). 
at $10,30 \mathrm{~min}, 1,3,6,12$, and $24 \mathrm{~h}$. Crystalline phases of the obtained samples were characterized by powder XRD. In our previous study, mixtures of Pure-OCP and OCPC were obtained under various conditions and the formation process of a singlephase of OCPC was discussed. ${ }^{16)}$ However, the formation processes of mixtures of Pure-OCP and OCPC were unclear. When excessive amounts of dicarboxylic acid with respect to stoichiometric composition of OCPC were used, the fraction of OCPC in the obtained samples would be increased with increasing reaction periods by transformation from Pure-OCP to OCPC. Hence, we investigated the formation process of a mixture of Pure-OCP and OCPC.

Hereafter, the samples are denoted as $1, x \mathrm{BDCA} y$, where $x$ indicates the position of the carboxyl group on the benzene ring $(x=2,3$, or 4$)$ and $y$ indicates the amounts of BDCAs (mmol) used for synthesis of OCPCs $(y=0.5,1,2,5,10$, or 25). For example, the 1,2BDCA2 indicates that the sample was synthesized by using $2.0 \mathrm{mmol}$ of 1,2BDCA. A sample synthesized in the absence of BDCAs was designated as the Control sample.

\section{Results}

Figure 3 shows powder XRD patterns of samples synthesized under conditions with various concentrations of 1,2BDCA. We used powder diffraction files (PDF) \#01-074-1301 and \#00-0471743 for identification of Pure-OCP and calcite, respectively. In the XRD patterns of the Control sample, 1,2BDCA0.5, 1,2BDCA1, and 1,2BDCA2, diffraction peaks assigned to OCP and calcite were detected. Diffraction peaks assigned to OCP were detected in 1,2BDCA5. In 1,2BDCA10 and 1,2BDCA25, diffraction peaks assigned to OCP and an unknown compound were detected. The intensities of the diffraction peaks assigned to the unknown compound increased with increasing amounts of added 1,2BDCA (phthalic acid) that was used for synthesis of OCP in 1,2BDCA10 and 1,2BDCA25. To identify the unknown compound, we synthesized a precipitate by mixing calcium chloride powder and phthalic acid solution. The $\mathrm{pH}$ of the phthalic acid solution was adjusted to 5.5 by ammonia solution. The precipitate provided the same diffraction patterns as the unknown compound shown in Fig. 3. Hence the unknown compound formed in $1,2 \mathrm{BDCA} 10$ and $1,2 \mathrm{BDCA} 25$ was probably calcium phthalate. According to Fig. 3, 100 reflection peaks of OCP were observed at around $2 \theta=4.7^{\circ}$ in all the samples. The peaks were slightly shifted to a lower angle in 1,2BDCA5,
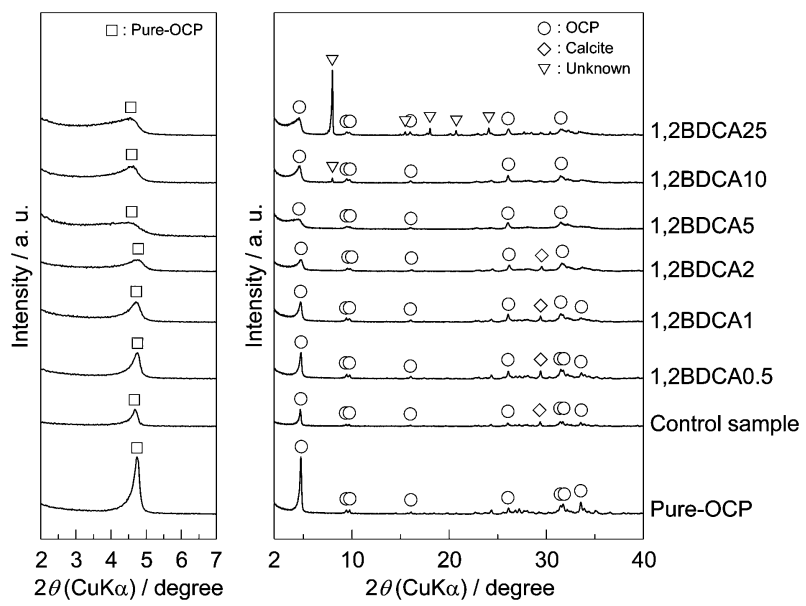

Fig. 3. Powder XRD patterns of samples synthesized under various concentrations of 1,2-denzenedicarboxylic acid (1,2BDCA).
1,2BDCA10, and 1,2BDCA25. Specifically, the peak was detected at $2 \theta=4.56^{\circ}$ in $1,2 \mathrm{BDCA} 25$, while the peak was detected at $2 \theta=4.74^{\circ}$ in Pure-OCP. The 100 reflection peaks in 1,2BDCA2, 1,2BDCA5, 1,2BDCA10, and 1,2BDCA25 were broader than that in the Control sample.

Figure 4 shows powder XRD patterns of samples synthesized under conditions with various concentrations of 1,3BDCA. In the XRD patterns of the Control sample, 1,3BDCA0.5, 1,3BDCA1, 1,3BDCA2, and 1,3BDCA5, diffraction peaks assigned to OCP and calcite were detected. In 1,3BDCA10 and 1,3BDCA25, diffraction peaks assigned to OCP were detected. According to Fig. 4, the peaks detected at $2 \theta=4.7^{\circ}$ in the Control sample, 1,3BDCA0.5, 1,3BDCA1, 1,3BDCA2, 1,3BDCA5, and $1,3 \mathrm{BDCA} 10$ were assigned to 100 reflection of Pure-OCP. Diffraction peaks were also detected at $2 \theta=3.9^{\circ}$ in $1,3 \mathrm{BDCA} 2$, 1,3BDCA5, 1,3BDCA10, and 1,3BDCA25. The interplanar spacing calculated from the diffraction peak detected at $2 \theta=$ $3.9^{\circ}$ was $2.3 \mathrm{~nm}$. Monma reported that the $d_{100}$ value of OCPC with $1,3 \mathrm{BDCA}(1,3 \mathrm{BDCA}-\mathrm{OCPC})$ is $\left.2.30 \mathrm{~nm} .{ }^{15}\right)$ Hence, the diffraction peak detected at $2 \theta=3.9^{\circ}\left(d_{100}=2.3 \mathrm{~nm}\right)$ was assignable to $1,3 \mathrm{BDCA}-\mathrm{OCPC}$. The $\mathrm{Ca} / \mathrm{P}$ molar ratio of the obtained 1,3BDCA-OCPC formed in 1,3BDCA25 was 1.56 .

Figure 5 shows powder XRD patterns of samples synthesized under the presence of various concentrations of 1,4BDCA. Diffraction peaks assigned to OCP and calcite were detected in the Control sample, 1,4BDCA0.5, and 1,4BDCA1. Diffraction peaks assigned to OCP, calcite, and calcium terephthalate trihydrate were detected in 1,4BDCA2 and 1,4BDCA5. We used PDF \#00-046-1873 for assignment of calcium terephthalate trihydrate. The intensities of the diffraction peaks assigned to calcium terephthalate trihydrate were increased with increasing added amounts of 1,4BDCA (terephthalic acid). Diffraction peaks assigned to OCP and calcium terephthalate trihydrate were detected in 1,4BDCA10. In 1,4BDCA25, diffraction peaks assigned to calcium terephthalate trihydrate and an unknown compound were detected. According to Fig. 5, a peak shift of 100 reflection of OCP was not detected in the samples synthesized under conditions with the presence of 1,4BDCA.

Chemical structures of the samples were characterized by FTIR. Figure 6 shows FTIR spectra of Pure-OCP, 1,2BDCA25 and 1,3BDCA25. We conducted assignment of the absorption peaks of OCPs based on the previous report. ${ }^{18)}$ In Pure-OCP, absorption peaks at 1193 , around 1120 , around 1034,601 , and
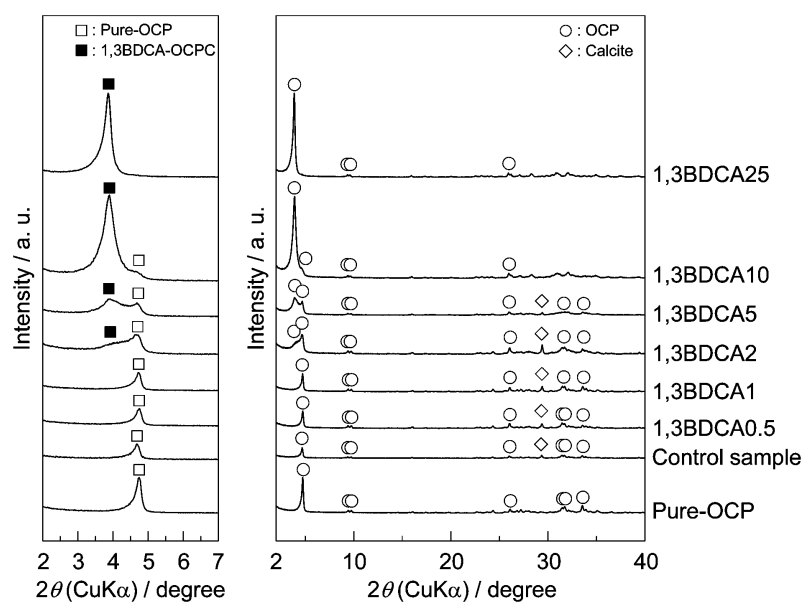

Fig. 4. Powder XRD patterns of samples synthesized under various concentrations of 1,3-denzenedicarboxylic acid (1,3BDCA). 

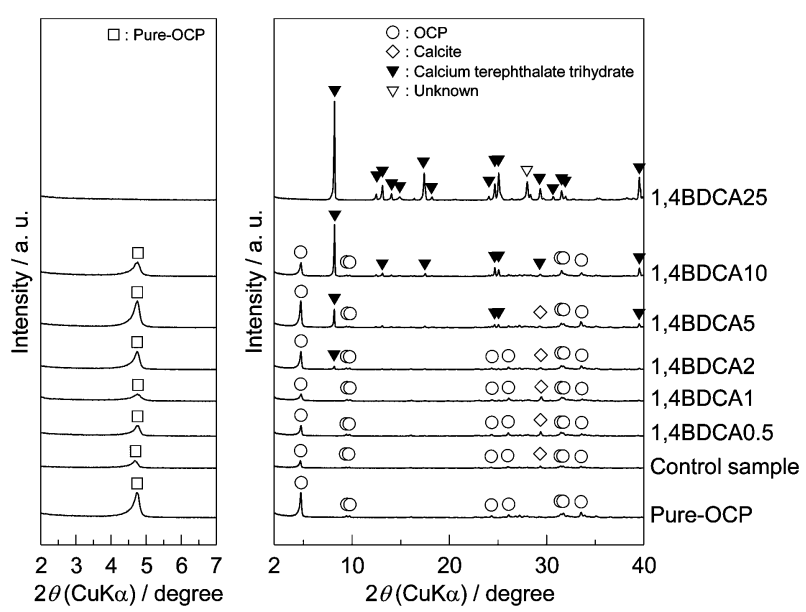

Fig. 5. Powder XRD patterns of samples synthesized under various concentrations of 1,4-denzenedicarboxylic acid (1,4BDCA).

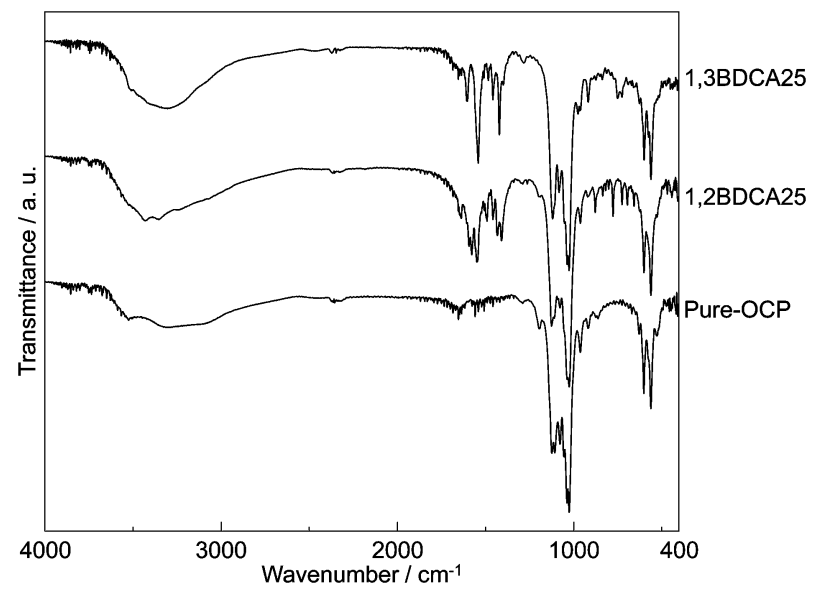

Fig. 6. FTIR spectra of Pure-OCP, 1,2BDCA25 and 1,3BDCA25.

$560 \mathrm{~cm}^{-1}$ were assigned to $\mathrm{OH}$-in plane-bending mode of $\mathrm{HPO}_{4}$ in the hydrated layer, $\mathrm{HPO}_{4}$ stretching mode, $\mathrm{PO}_{4}$ stretching mode, $\mathrm{PO}_{4}$ bending mode, and $\mathrm{PO}_{4}$ bending mode, respectively. The absorption peaks at 1193 , around 1120 , around 1034,601 , and $560 \mathrm{~cm}^{-1}$ were detected in 1,2BDCA25. The intensity of absorption peak at $1193 \mathrm{~cm}^{-1}$ in 1,2BDCA25 was weaker than that in Pure-OCP. According to X-ray diffraction patterns of Fig. 3, 1,2BDCA25 was mixture of OCP and an unknown compound, which might be calcium phthalate. Absorption peaks detected at ranged $1660-1420 \mathrm{~cm}^{-1}$ would be derived from the carboxyl group of calcium phthalate. The absorption peaks at around 1120 , around 1034,601 , and $560 \mathrm{~cm}^{-1}$ were detected in $1,3 \mathrm{BDCA} 25$. The absorption peak at $1193 \mathrm{~cm}^{-1}$ was detected in Pure-OCP, but was not detected in 1,3BDCA25. In the case of OCPC with succinate, adipate, suberate, sebacate, fumarate, and citrate ions, absorption peaks derived from the carboxyl group of the dicaboxylate ion incorporated into OCP were detected at $1586-1548$ and $1470-1430 \mathrm{~cm}^{-1}$. Therefore, the absorption peaks detected at these ranges in 1,3BDCA25 were derived from carboxyl groups of 1,3BDCA. In general, out-of-plane bending vibrations of $\mathrm{C}-\mathrm{H}$ in 1,3-disubstituted benzene are detected at $820-780$ and $730-680 \mathrm{~cm}^{-1} \cdot{ }^{19)}$ Hence the two peaks detected around these ranges were probably derived from the out-of-plane bending vibrations of $\mathrm{C}-\mathrm{H}$ in the benzene ring.
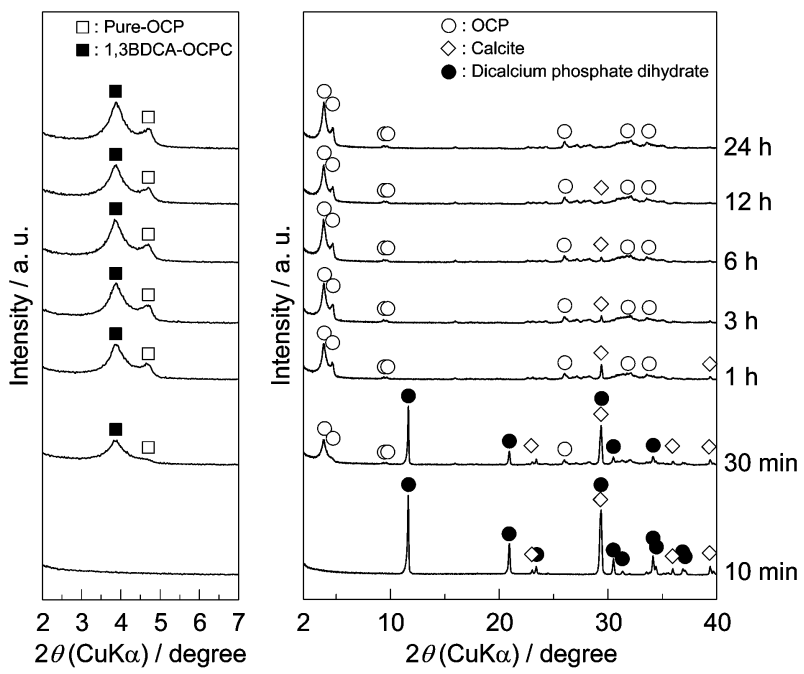

Fig. 7. Time dependence of the crystalline phases in 1,3BDCA5.

The time dependence of the crystalline phases in 1,3BDCA5 was characterized by powder XRD to investigate the formation process of the mixture of 1,3BDCA-OCPC and Pure-OCP. XRD patterns of precipitates formed in 1,3BDCA5 at 10,30 min, 1, 3, 6,12 , and $24 \mathrm{~h}$ are shown in Fig. 7. In $10 \mathrm{~min}$, diffraction peaks assigned to DCPD and calcite were detected. We used PDF \#00-011-0293 for assignment of DCPD. In $30 \mathrm{~min}$, diffraction peaks assigned to OCP, DCPD, and calcite were detected. From $1 \mathrm{~h}$ until $12 \mathrm{~h}$, diffraction peaks assigned to OCP and calcite were detected. While diffraction peak intensities of calcite were decreased with increasing reaction periods, those of OCP were almost constant. In $24 \mathrm{~h}$, diffraction peaks assigned to OCP were detected. According to Fig. 7, both Pure-OCP and 1,3BDCAOCPC were detected beginning at $30 \mathrm{~min}$.

Figure 8 shows SEM images of Pure-OCP, 1,3BDCA1, and 1,3BDCA25. The morphology of Pure-OCP was plate-like shaped with approximately $1 \mu \mathrm{m}$ in width and approximately $10 \mu \mathrm{m}$ in length. The shape and size of precipitates formed in 1,3BDCA1 were similar to Pure-OCP, while precipitates formed in $1,3 \mathrm{BDCA} 25$ had a wider shape than precipitates formed in $1,3 \mathrm{BDCA} 1$.

\section{Discussion}

When a dicarboxylic acid is incorporated into OCP, the $d_{100}$ value of OCP is increased. Hence the diffraction peak assigned to 100 reflection of OCP shifts to a lower angle by incorporation of dicarboxylate ion into OCP.

According to Fig. 3, the slight peak shift was detected in 1,2BDCA5, 1,2BDCA10, and 1,2BDCA25. The $d_{100}$ value of OCPC with $1,2 \mathrm{BDCA}(1,2 \mathrm{BDCA}-\mathrm{OCPC})$ is $2.30 \mathrm{~nm} .{ }^{15)}$ According to the $d_{100}$ value of $1,2 \mathrm{BDCA}-\mathrm{OCPC}$, we calculated that the 100 reflection peak of 1,2BDCA-OCPC is detected at $2 \theta=3.84^{\circ}$. The 100 reflection peak of $1,2 \mathrm{BDCA} 25$ is detected at $2 \theta=4.56^{\circ}$. Hence, 1,2BDCA-OCPC was not formed in samples synthesized under the conditions with 1,2BDCA addition. The 100 reflection peaks of 1,2BDCA5, 1,2BDCA10, and $1,2 \mathrm{BDCA} 25$ were detected at slightly lower angles than $2 \theta=4.7^{\circ}$. The FTIR spectrum of $1,2 \mathrm{BDCA} 25$ also suggested incorporation of 1,2BDCA into OCP (Fig. 6), because the intensity of absorption peak derived from $\mathrm{HPO}_{4}{ }^{2-}$ in hydrated layer at $1193 \mathrm{~cm}^{-1}$ in 1,3 BDCA25 was weaker than that in Pure-OCP. These findings indicate that $\mathrm{HPO}_{4}{ }^{2-}$ in the hydrated layer in OCP is partially substituted by $1,2 \mathrm{BDCA}$. 

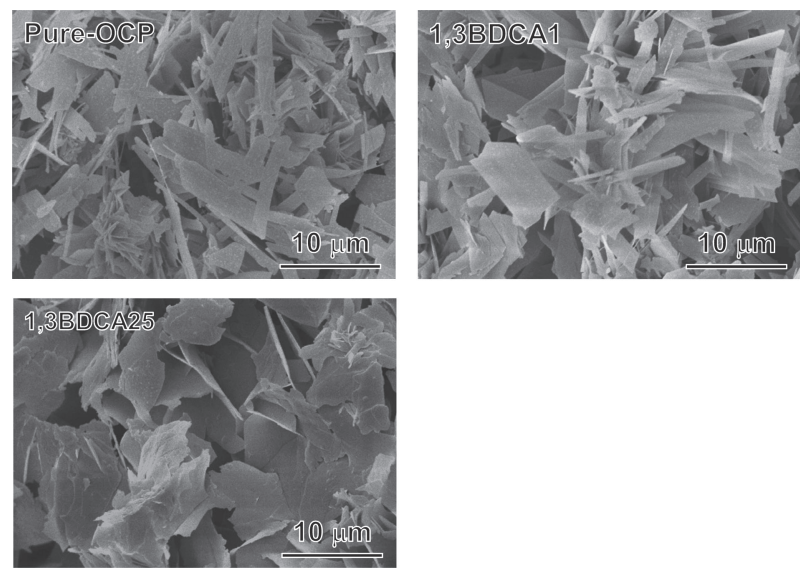

Fig. 8. SEM images of Pure-OCP, 1,3BDCA1, and 1,3BDCA25.

Addition of excessive amounts of 1,2BDCA had little advantage for the formation of 1,2BDCA-OCPC. Monma obtained $1,2 \mathrm{BDCA}-\mathrm{OCPC}$ by the hydrolysis of $\alpha$-TCP in $0.1 \mathrm{~mol} \cdot \mathrm{dm}^{-3}$ (M) of 1,2BDCA solution with an initial $\mathrm{pH} 6.0$ at $40^{\circ} \mathrm{C}^{15)} \mathrm{We}$ did not obtained 1,2BDCA-OCPC by mixing $\mathrm{CaCO}_{3}$ and $\mathrm{H}_{3} \mathrm{PO}_{4}$ in approximately $0-0.25 \mathrm{M}$ of $1,2 \mathrm{BDCA}$ solutions with an initial $\mathrm{pH} 5.5$ at $60^{\circ} \mathrm{C}$. It is difficult to explain the reasons for the difference in incorporation of 1,2BDCA, since many experimental parameters (e.g. concentration of 1,2BDCA, kind of precursor, and reaction temperature) were different between this study and Monma's study, but we briefly discuss the effect of some experimental parameters on incorporation of 1,2BDCA as follows. Based on our previous report, concentration of dicarboxylic acid is one of the dominant factors of formation of OCPC. $^{16)} 1,2 \mathrm{BDCA}$ concentration in 1,2BDCA25, which was approximately $0.25 \mathrm{M}$, was larger than that in Monma's synthesis method, and hence we used enough amounts of 1,2BDCA for incorporation of 1,2BDCA into OCP. The precursor for OCPC was DCPD in our synthesis method, while it was $\alpha$-TCP in Monma's synthesis method. The solubility of DCPD is lower than that of $\alpha$-TCP with a pH less than 7.5.20) The solubilities of the precursors may be a factor for OCPC formation, because the reaction rate depends on the solubilities of the precursors. The initial $\mathrm{pH}$ values of the reaction mixture in our synthesis method were almost the same as those in Monma's synthesis method. However the $\mathrm{pH}$ values of the reaction mixture should be changed during the formation process of OCPs and the time dependence of the $\mathrm{pH}$ values between our synthesis method and Monma's synthesis method should be different. The $\mathrm{pH}$ in the reaction mixture affects the reaction rate of the precursors, the solubility of the precursors and OCPC, the degree of supersaturation with respect to $\mathrm{OCPC}$, and the dissociation states of phosphoric acid and 1,2BDCA. Hence $\mathrm{pH}$ values in the reaction mixture may affect the incorporation of 1,2BDCA into OCP. The reaction temperature in our synthesis method was $60^{\circ} \mathrm{C}$, while that in Monma's synthesis method was $40^{\circ} \mathrm{C}$. Reaction temperature affects the reaction rate, the solubilities of the precursors and OCPC, the degree of supersaturation with respect to OCPC, and the dissociation constants of phosphoric acid and 1,2BDCA. Hence reaction temperature may affect incorporation of 1,2BDCA into OCP. In the future, we will attempt to reveal the reasons from the viewpoints of inorganic synthesis, and solution and coordination chemistry, because we considered that the following parameters dominate formation of OCPCs: $\mathrm{pH}$, concentrations of calcium and phosphate ions in the reaction solutions, reaction temperature, reaction rate of precursors, and complex formation of dicarboxylate ions with calcium ions.

According to Fig. 4, 1,3BDCA-OCPC was formed in 1,3BDCA2, 1,3BDCA5, 1,3BDCA10, and 1,3BDCA25. The FTIR spectrum of 1,3BDCA25 also indicated incorporation of 1,3BDCA into OCP (Fig. 6). The absorption peak of $\mathrm{HPO}_{4}{ }^{2-}$ at $1193 \mathrm{~cm}^{-1}$ was not detected in $1,3 \mathrm{BDCA} 25$ because of the substitution of $\mathrm{HPO}_{4}{ }^{2-}$ in the hydrated layer by $1,3 \mathrm{BDCA}$. $1,3 \mathrm{BDCA}-\mathrm{OCPC}$ was formed in conditions with an addition of 1,3BDCA larger than $2 \mathrm{mmol}$. The 1,3BDCA-OCPC was formed as a single phase in 1,3BDCA25. These findings indicated that excessive amounts of dicarboxylic acids were required for formation of 1,3BDCA-OCPC. The $\mathrm{Ca} / \mathrm{P}$ molar ratio of the 1,3BDCA-OCPC formed in 1,3BDCA25 was 1.56 . Hence the composition of the obtained 1,3BDCA-OCPC was $\mathrm{Ca}_{8}\left(\mathrm{HPO}_{4}\right)_{1.13}(\mathrm{DCI})_{0.87}\left(\mathrm{PO}_{4}\right)_{4} \cdot m \mathrm{H}_{2} \mathrm{O}$.

In Fig. 5, the peak shift of the 100 reflection of OCP did not occur, suggesting that 1,4BDCA was not incorporated into OCP. This finding was consistent with the previous report. ${ }^{15)}$ The crystalline phases of precipitates were changed from Pure-OCP to calcium terephthalate trihydrate with increasing amounts of added 1,4BDCA. Hence, OCPC with 1,4BDCA hardly formed even though the amount of added 1,4BDCA increased.

When the dicarboxylate ion was incorporated into OCP, no calcium dicarboxylate salt was formed (Fig. 4). Conversely, when the dicarboxylate ion was not incorporated into OCP, the calcium dicarboxylate salt was formed (Figs. 3 and 5). Hence the solubility of calcium dicarboxylate salts would be a dominant factor for formation of BDCA-OCPC. When we used 1,2BDCA and 1,4BDCA for synthesis of OCPCs, calcium dicarboxylate salts were formed. Additionally, the amounts of the calcium dicarboxylate salts were increased with increasing amounts of added dicarboxylic acids. Concentrations of 1,2BDCA and $1,4 \mathrm{BDCA}$, as well as calcium ion, in the reaction mixture were decreased by formation of the calcium dicarboxylate salts. The driving force for the formation of OCPCs was decreased by the formation of the calcium dicarboxylate salts, and hence OCPC with 1,2BDCA and 1,4BDCA were not formed. By contrast, when we used 1,3BDCA for synthesis of OCPC, no calcium dicarboxylate salt was formed. As a result of the increase in driving force for the formation of OCPCs with increasing amounts of added 1,3BDCA, 1,3BDCA-OCPC was formed.

We attempted semi-quantitation of the amounts of Pure-OCP and BDCAs-OCPC by using the intensities of the 100 reflection peaks of Pure-OCP and BDCAs-OCPC. The 100 reflections of Pure-OCP and 1,3BDCA-OCPC were detected at $2 \theta=4.7$ and $3.9^{\circ}$, respectively. $F_{\mathrm{OCPC}}$, which shows the semi-quantitative fraction of OCPC, was defined by the following equation.

$$
F_{\mathrm{OCPC}}=I_{\mathrm{OCPC}} /\left(I_{\text {Pure-CP }}+I_{\mathrm{OCPC}}\right)
$$

Where $I_{\text {Pure-OCP }}$ and $I_{\mathrm{OCPC}}$ are the estimated intensities of the 100 reflection peak assigned to Pure-OCP and OCPC, respectively. Figure 9 shows the relationship between $F_{\mathrm{OCPC}}$ and the amounts of added dicarboxylic acids. When the amounts of added 1,3BDCA were $0-1.0 \mathrm{mmol}, F_{1,3 \mathrm{BDCA}-\mathrm{OCPC}}$ was almost zero. $F_{1,3 \mathrm{BDCA}-\mathrm{OCPC}}$ was drastically increased with increasing amounts of added 1,3BDCA when the amounts of added 1,3BDCA were $2.0-10 \mathrm{mmol}$. When the amounts of added $1,3 \mathrm{BDCA}$ were $10-25 \mathrm{mmol}$, the $F_{1,3 \mathrm{BDCA}-\mathrm{OCPC}}$ values gradually increased. When the amount of added 1,3BDCA was $25 \mathrm{mmol}$, $F_{1,3 \mathrm{BDCA}-\mathrm{OCPC}}$ was almost equal to 1.0 . The stoichiometric composition of OCPC is $\mathrm{Ca}_{8}\left(\mathrm{HPO}_{4}\right)(\mathrm{DCI})\left(\mathrm{PO}_{4}\right)_{4} \cdot m \mathrm{H}_{2} \mathrm{O}$. The molar ratio of calcium ions to phosphate ions to dicarboxylate ions is 


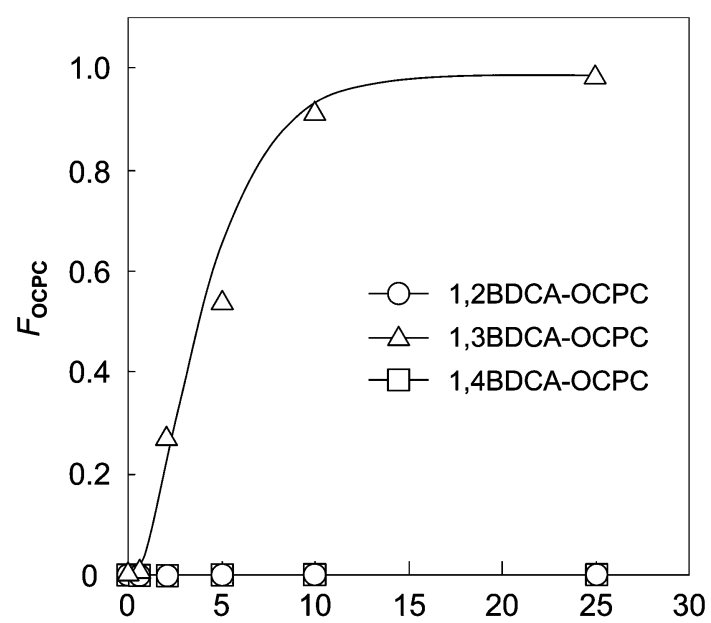

Amounts of added dicarboxylic acid / mmol

Fig. 9. Relationship between $F_{\mathrm{OCPC}}$ and the amounts of added dicarboxylic acids. The $F_{\mathrm{OCPC}}$ is the semi-quantitative fraction of OCPC in the samples. The $F_{\mathrm{OCPC}}$ values were calculated from the intensities of the 100 reflections of Pure-OCP ( $\left.I_{\text {Pure-OCP }}\right)$ and OCPCs $\left(I_{\text {OCPC }}\right)$.

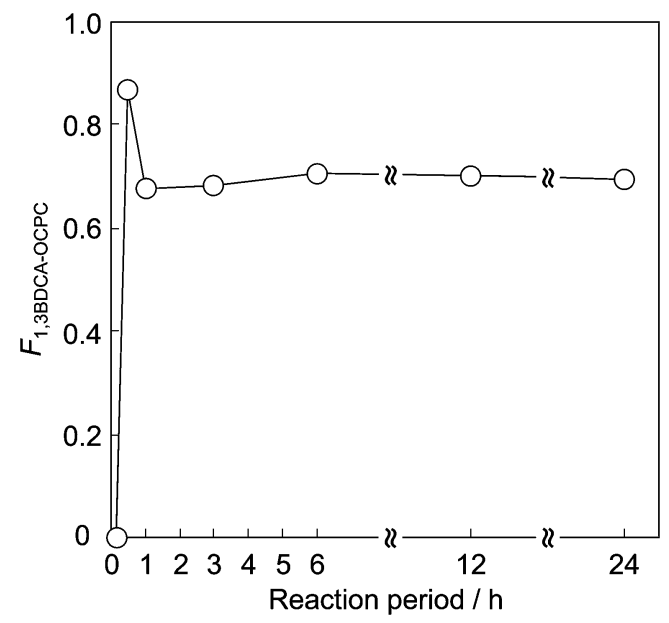

Fig. 10. Time dependence of the $F_{1,3 \mathrm{BDCA}-\mathrm{OCPC}}$ values in 1,3BDCA5.

thus 8:5:1. We used $8.0 \mathrm{mmol}$ of $\mathrm{CaCO}_{3}$ and $5.0 \mathrm{mmol}$ of $\mathrm{H}_{3} \mathrm{PO}_{4}$, hence $1.0 \mathrm{mmol}$ of BDCAs was the stoichiometric amount for OCPC. Addition of 1,3BDCA larger than 25 times with respect to the stoichiometric composition for OCPC was required for formation of 1,3BDCA-OCPC as a single phase. These findings implied that addition of an excessive amount of 1,3BDCA prevented formation of Pure-OCP and had an advantage in the formation of 1,3BDCA-OCPC. In contrast, 1,2BDCA-OCPC and $\mathrm{OCPC}$ with 1,4BDCA (1,4BDCA-OCPC) were not formed, hence $F_{1,2 \mathrm{BDCA}-\mathrm{OCPC}}$ and $F_{1,4 \mathrm{BDCA}-\mathrm{OCPC}}$ values were zero in Fig. 9.

OCPC with BDCA was formed with the addition of 1,3BDCA. The time dependent changes of $F_{1,3 \mathrm{BDCA}-\mathrm{OCPC}}$ values were calculated from the XRD patterns of 1,3BDCA5 shown in Fig. 7. Figure 10 shows time dependence of the $F_{1,3 \mathrm{BDCA}-\mathrm{OCPC}}$ values of $1,3 \mathrm{BDCA} 5$. In $30 \mathrm{~min}$, the $F_{1,3 \mathrm{BDCA}-\mathrm{OCPC}}$ value was 0.87 . In $1 \mathrm{~h}$, the $F_{1,3 \mathrm{BDCA}-\mathrm{OCPC}}$ value was 0.67 . The decrease in the

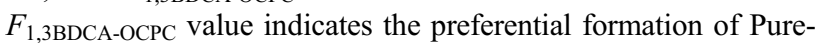
OCP during the reaction periods from $30 \mathrm{~min}$ to $1 \mathrm{~h}$. According to Fig. $9, F_{1,3 \mathrm{BDCA}-\mathrm{OCPC}}$ values were dramatically changed by amounts of added 1,3BDCA around $5.0 \mathrm{mmol}$. Hence PureOCP was preferentially formed as a result of a decrease in the formation rate of 1,3BDCA-OCPC by decreasing the 1,3BDCA concentration in the reaction solution due to 1,3BDCA-OCPC formation. It is reasonable to interpret that the Pure-OCP was formed from DCPD rather than 1,3BDCA-OCPC during the reaction periods from $30 \mathrm{~min}$ to $1 \mathrm{~h}$ because of the following two reasons. One, according to the synthesis method of OCPC by using $\mathrm{CaCO}_{3}$ and $\mathrm{H}_{3} \mathrm{PO}_{4}$, OCPC were formed from DCPD. ${ }^{15), 16)}$ Two, $F_{1,3 \mathrm{BDCA}-\mathrm{OCPC}}$ values were almost constant at 0.7 beginning at $1 \mathrm{~h}$, hence transformation from 1,3BDCA-OCPC to Pure-OCP as well as Pure-OCP to 1,3BDCA-OCPC hardly occurred.

In $1,3 \mathrm{BDCA} 1$, the crystalline phase of precipitates was PureOCP (Fig. 4). Precipitates formed in 1,3BDCA25 were larger and wider than precipitates formed in 1,3BDCA1 and Pure-OCP, as shown in Fig. 8. OCP synthesized through a wet process displays mainly the (100) face, ${ }^{21), 22)}$ which is calcium ion-rich. ${ }^{23)}$ Hence, 1,3BDCA electrostatically interacted with calcium ion and adsorbed on the (100) face. The crystal growth rate in the [100] direction should decrease by adsorption of 1,3BDCA. As a result, the crystals tended to expose more (100) face in the presence of 1,3BDCA than in the absence of 1,3BDCA. The activity of calcium ions was decreased by chelate formation, hence the degree of supersaturation with respect to OCPC was reduced. The nucleation frequency of OCPC was decreased, and crystal growth of OCPC could occur preferentially. Thus crystal sizes were increased by the addition of 1,3BDCA.

\section{Conclusions}

We investigated the formation of octacalcium phosphate (OCP) with incorporated benzenedicarboxylic acids, namely 1,2-benzenedicarboxylic acid (1,2BDCA, phthalic acid), 1,3-benzenedicarboxylic acid (1,3BDCA, isophthalic acid), and 1,4-benzenedicarboxylic acid (1,4BDCA, terephthalic acid). We used calcium carbonate, phosphoric acid and various amounts of BDCAs as starting materials. OCP carboxylate (OCPC) with 1,3BDCA (1,3BDCA-OCPC) was formed, while $1,4 \mathrm{BDCA}$ was not incorporated into OCP. In the case of 1,2BDCA, a small amount of $1,2 \mathrm{BDCA}$ was incorporated into OCP. Under most conditions, OCPC with 1,3BDCA (1,3BDCA-OCPC) was formed with dicarboxylate-free OCP (Pure-OCP). The fractions of 1,3BDCAOCPC with respect to Pure-OCP in the samples were increased with increasing amounts of 1,3BDCA. Finally, we obtained $1,3 \mathrm{BDCA}-\mathrm{OCPC}$ as a single phase, when the added amount of $1,3 \mathrm{BDCA}$ was $25 \mathrm{mmol}$, which is 25 times larger than the stoichiometric composition for OCPC. Addition of excessive amounts of 1,3BDCA prevents formation of Pure-OCP and has an advantage in the formation of 1,3BDCA-OCPC.

Acknowledgments This work was partially supported by a Grant-in-Aid for JSPS Fellows (23.2593) and a Grant-in-Aid for Scientific Research (No. 22107007) on the Innovative Areas: "Fusion Materials" (Area no. 2206) from the Ministry of Education, Culture, Sports, Science and Technology (MEXT), Japan.

\section{References}

1) S. Takagi, L. C. Chow and K. Ishikawa, Biomaterials, 19, 1593-1599 (1998).

2) M. Kitamura, C. Ohtsuki, S. Ogata, M. Kamitakahara and M. Tanihara, J. Ceram. Soc. Japan, 112, S817-S921 (2004).

3) T. Okuda, K. Ioku, I. Yonezawa, H. Minagi, Y. Gonda, G. Kawachi, M. Kamitakahara, Y. Shibata, H. Murayama, H. Kurosawa and T. Ikeda, Biomaterials, 29, 2719-2728 (2008).

4) T. Goto, I. Y. Kim, K. Kikuta and C. Ohtsuki, Ceram. Int., 38, 
1003-1010 (2012).

5) T. Goto, I. Y. Kim, K. Kikuta and C. Ohtsuki, J. Ceram. Soc. Japan, 120, 131-137 (2012).

6) J. C. Elliott, "Structure and chemistry of the apatite and other calcium orthophosphate", Elsevier, Amsterdam (1994) pp. 2426,32 .

7) W. E. Brown, J. P. Smith, J. R. Lehr and A. W. Frazier, Nature, 196, 1048-1055 (1962).

8) H. Monma and M. Goto, Bull. Chem. Soc. Japan., 56, 38433844 (1983).

9) H. Monma, Bull. Chem. Soc. Japan., 57, 599-600 (1984).

10) S. Aoki, A. Nakahira, H. Nakayama, K. Sakamoto, S. Yamaguchi and K. Suganuma, J. Phys. Chem. Solids, 65, 465-470 (2004).

11) M. Marković, B. O. Fowler and W. E. Brown, Chem. Mater., 5, 1401-1405 (1993).

12) S. Aoki, K. Sakamoto, S. Yamaguchi and A. Nakahira, J. Ceram. Soc. Japan, 108, 909-914 (2000) [in Japanese].

13) A. Nakahira, S. Aoki, K. Sakamoto and S. Yamaguchi, J. Mater. Sci.: Mater. Med., 12, 793-800 (2001).
14) H. Monma, Gypsum \& Lime, 237, 108-114 (1992) [in Japanese].

15) M. Kamitakahara, H. Okano, M. Tanihara and C. Ohtsuki, J. Ceram. Soc. Japan, 116, 481-485 (2008).

16) T. Yokoi, H. Kato, I. Y. Kim, K. Kikuta, M. Kawashita and C. Ohtsuki, Ceram. Int., 38, 3815-3820 (2012).

17) S. R. Freeman, F. Jones, M. I. Ogden, A. Oliviera and W. R. Richmond, Cryst. Growth Des., 6, 2579-2587 (2006).

18) M. Marković, B. O. Fowler and W. E. Brown, Chem. Mater., 5, 1406-1416 (1993).

19) B. Stuart, "Modern Infrared Spectroscopy", Ed. by D. J. Ando, John Wiley \& Sons, England (1996) pp. 101-103.

20) L. C. Cho, J. Ceram. Soc. Japan, 99, 954-964 (1991).

21) M. Iijima, H. Tohda and Y. Moriwaki, J. Cryst. Growth, 116, 319-326 (1992).

22) A. Bigi, E. Boanini, G. Cojazzi, G. Falini and S. Panzavolta, Cryst. Growth Des., 1, 239-244 (2001).

23) K. Ohta, H. Monma, S. Takahashi and H. Kobayashi, J. Ceram. Soc. Japan, 107, 577-581 (1999) [in Japanese]. 